\title{
Gender influences left ventricular remodelling in the setting of aortic stenosis but does not appear to impact on reverse remodelling following transcatheter aortic valve implantation
}

\author{
Laura E Dobson ${ }^{1 *}$, Tarique A Musa ${ }^{1}$, Akhlaque Uddin', Timothy A Fairbairn', Daniel J Blackman², David P Ripley ${ }^{1}$, \\ Adam K McDiarmid ${ }^{1}$, Peter P Swoboda ${ }^{1}$, Bara Erhayiem¹, Pankaj Garg' ${ }^{1}$, Sven Plein ${ }^{1}$, John P Greenwood ${ }^{1}$
}

From 18th Annual SCMR Scientific Sessions

Nice, France. 4-7 February 2015

\section{Background}

Left ventricular (LV) remodelling in the setting of the chronic pressure overload of aortic stenosis (AS) appears to differ according to gender. Women appear to have an improved survival compared to men following transcatheter aortic valve implantation (TAVI) but the reasons for this are yet to be established. Potential mechanisms include differing patterns of LV reverse remodelling, LV fibrosis regression and reduced aortic regurgitation in females. We sought to establish using cardiac magnetic resonance (CMR) imaging, the reference standard noninvasive technique for LV mass and scar quantification, whether there were any gender differences in these parameters before and after TAVI.

\section{Methods}

53 patients with symptomatic severe aortic stenosis undergoing TAVI were prospectively enrolled between April 2009 and March 2014. Patients with contraindications to CMR were excluded and all patients provided informed written consent. All patients underwent an identical 1.5T CMR protocol (Intera, Philips) prior to and at a median of 6 months following TAVI (IQR 5-6 months). Multi slice, multiphase imaging was carried out using a standard steady-state free procession pulse sequence in the short axis to cover the entire left ventricle. Late gadolinium enhancement imaging was performed $10 \mathrm{~min}$ after the administration of $0.2 \mathrm{mmol} / \mathrm{kg}$ of gadoteric acid (Doteram,

${ }^{1}$ Multidisciplinary Cardiovascular Reseacrh Centre \& Leeds Institute for Cardiovascular and Metabolic Medicine, University of Leeds, Leeds, UK Full list of author information is available at the end of the article
Table 1 Basic clinical, echocardiographic and procedural characteristics. Data expressed as mean \pm SD unless otherwise stated.

\begin{tabular}{|c|c|c|}
\hline & Male & Female \\
\hline Number of patients, (\%) & $30(57)$ & 43 \\
\hline Age & $78 \pm 6$ & $84 \pm 8$ \\
\hline STS Mortality score, \% & $4.2 \pm 2.4$ & $7.2 \pm 3.6$ \\
\hline STS Morbidity/mortality score, $\%$ & $23.8 \pm 8.1$ & $28.1 \pm 8.8$ \\
\hline Hypertension, n (\%) & $10(33)$ & $14(61)$ \\
\hline \multicolumn{3}{|l|}{ Echocardiographic characteristics } \\
\hline AVAi $(\mathrm{cm} / \mathrm{m} 2)$ & $0.32 \pm 0.1$ & $0.32 \pm 0.08$ \\
\hline Peak pressure drop & $86.3 \pm 22$ & $93.8 \pm 21.6$ \\
\hline \multicolumn{3}{|l|}{ Procedural characteristics } \\
\hline \multicolumn{3}{|l|}{ TAVI Type, n (\%) } \\
\hline Medtronic Corevalve & $22(73)$ & $19(83)$ \\
\hline Boston Lotus & $8(27)$ & $3(13)$ \\
\hline Medtronic Engager & $0(0)$ & $1(4)$ \\
\hline \multicolumn{3}{|l|}{ TAVI size, $\mathrm{n}(\%)$} \\
\hline $23 \mathrm{~mm}$ & $2(7)$ & $4(17)$ \\
\hline $26 \mathrm{~mm}$ & $2(7)$ & $7(30)$ \\
\hline $27 \mathrm{~mm}$ & $7(23)$ & $1(4)$ \\
\hline $29 \mathrm{~mm}$ & $17(56)$ & $11(49)$ \\
\hline $31 \mathrm{~mm}$ & $2(7)$ & $0(0)$ \\
\hline \multicolumn{3}{|l|}{ Access site, $\mathrm{n}(\%)$} \\
\hline Femoral & $26(65)$ & $21(92)$ \\
\hline Subclavian & $3(10)$ & $1(4)$ \\
\hline Apical & $0(0)$ & $1(4)$ \\
\hline Direct aortic & $1(4)$ & $0(0)$ \\
\hline
\end{tabular}

STS: Society of Thoracic Surgeons 
Table 2 Pre and 6 month post-TAVI values for male and female gender. Values expressed as mean \pm SD.

\begin{tabular}{|c|c|c|c|c|}
\hline & Pre-TAVI & Post-TAVI & Change & P Value \\
\hline \multicolumn{5}{|c|}{ LV Ejection fraction (\%) } \\
\hline Men & $52.6 \pm 13.8$ & $54.7 \pm 11.3$ & $2.1 \pm 8.1$ & 0.18 \\
\hline Women & $57.9 \pm 11.4$ & $59.5 \pm 11.8$ & $1.5 \pm 6.5$ & 0.28 \\
\hline$P$ Value & 0.15 & 0.15 & 0.79 & \\
\hline \multicolumn{5}{|c|}{ LV End diastolic volume indexed (ml/m2) } \\
\hline Men & $105.0 \pm 27.7$ & $97.2 \pm 21.9$ & $7.8 \pm 20$ & 0.04 \\
\hline Women & $90.2 \pm 17.0$ & $81.8 \pm 19.3$ & $8.4 \pm 15.8$ & 0.02 \\
\hline$P$ Value & 0.03 & 0.01 & 0.92 & \\
\hline \multicolumn{5}{|c|}{ LV Mass indexed (g/m2) } \\
\hline Men & $81.8 \pm 21.6$ & $64.0 \pm 17.9$ & $17.7 \pm 9.1$ & $<0.001$ \\
\hline Women & $69.6 \pm 18.8$ & $53.9 \pm 14.4$ & $15.7 \pm 8.4$ & $<0.001$ \\
\hline P Value & 0.04 & 0.03 & 0.43 & \\
\hline \multicolumn{5}{|c|}{ LV scar (g) (FWHM method) } \\
\hline Men & $5.0 \pm 5.7$ & $5.1 \pm 6.2$ & $0.1 \pm 2.1$ & 0.88 \\
\hline Women & $1.7 \pm 2.5$ & $1.3 \pm 2.6$ & $0.6 \pm 1.4$ & 0.25 \\
\hline$P$ Value & 0.01 & 0.01 & 0.36 & \\
\hline \multicolumn{5}{|c|}{ Left atrial volume indexed (ml/m2) } \\
\hline Men & $74.2 \pm 23.5$ & $65.5 \pm 23.3$ & $8.7 \pm 13.7$ & 0.002 \\
\hline Women & $63.7 \pm 17.2$ & $58.3 \pm 19.3$ & $5.4 \pm 31.0$ & 0.2 \\
\hline$P$ Value & 0.08 & 0.25 & 0.47 & \\
\hline \multicolumn{5}{|c|}{ Aortic regurgitant fraction (\%) } \\
\hline Men & $12.8 \pm 8.2$ & $6.5 \pm 6.5$ & $6.3 \pm 9.3$ & 0.001 \\
\hline Women & $9.7 \pm 9.5$ & $6.2 \pm 5.2$ & $3.0 \pm 10.5$ & 0.12 \\
\hline P Value & 0.22 & 0.87 & 0.25 & \\
\hline
\end{tabular}

FWHM: Full width half max

Guerbet, Villepinte). Quantitative analysis was performed using dedicated computer software $\left(\mathrm{CVI}^{42}\right.$, Circle Cardiovascular Imaging, Alberta, Canada).

\section{Results}

Basic clinical, echocardiographic and procedural characteristics can be seen in Table 1 . Women with severe AS have a smaller indexed LV mass (LVMi) (69.6 $\pm 18.8 \mathrm{~g} /$ $\mathrm{m}^{2}$ Vs $\left.81.8 \pm 21.6 \mathrm{~g} / \mathrm{m}^{2}, \mathrm{p}=0.03\right)$ less baseline myocardial scar $(1.7 \pm 2.5 \mathrm{~g}$ Vs $5.0 \pm 5.7 \mathrm{~g}, \mathrm{p}=0.01)$ and a smaller indexed $\mathrm{LV}$ end diastolic volume (LVEDVi) than men $\left(90.2 \pm 17 \mathrm{ml} / \mathrm{m}^{2}\right.$ Vs. $\left.105 \pm 27.7 \mathrm{ml} / \mathrm{m}^{2} ; \mathrm{p}=0.03\right)$. A trend towards a smaller indexed left atrial volume (LAVi) $\left(63.7 \pm 17.2 \mathrm{ml} / \mathrm{m}^{2} \mathrm{Vs} 74.2 \pm 23.5 \mathrm{ml} / \mathrm{m}^{2}, \mathrm{p}=0.08\right)$ and better ejection fraction $(57.9 \pm 11.4 \%$ Vs $52.6 \pm 13.8 \%$, $\mathrm{p}=0.15)$ was also seen in women when compared with men. Six months following TAVI, there was no significant difference seen between genders with regards to LV mass regression, change in ejection fraction, change in myocardial scar burden, change in left atrial volume or change in aortic regurgitant fraction (Table 2).

\section{Conclusions}

In the setting of the chronic pressure overload of aortic stenosis, there appears to be a difference in LV remodelling between genders with a smaller LV cavity, less fibrosis and a lower LV mass in women compared with men. Six months following TAVI, there did not appear to be a significant difference in LV reverse remodelling, change in myocardial scar burden or aortic regurgitant fraction according to gender.

\section{Funding}

This study was part funded by the British Heart Foundation (BHF) (PG/11/126/29321).

\section{Authors' details}

${ }^{1}$ Multidisciplinary Cardiovascular Reseacrh Centre \& Leeds Institute for Cardiovascular and Metabolic Medicine, University of Leeds, Leeds, UK. ${ }^{2}$ Yorkshire Heart Centre, Leeds Teaching Hospitals Trust, Leeds, UK. 
doi:10.1186/1532-429X-17-S1-P332

Cite this article as: Dobson et al:: Gender influences left ventricular remodelling in the setting of aortic stenosis but does not appear to impact on reverse remodelling following transcatheter aortic valve implantation. Journal of Cardiovascular Magnetic Resonance 2015 17(Suppl 1):P332.

Submit your next manuscript to BioMed Central and take full advantage of:

- Convenient online submission

- Thorough peer review

- No space constraints or color figure charges

- Immediate publication on acceptance

- Inclusion in PubMed, CAS, Scopus and Google Scholar

- Research which is freely available for redistribution

Submit your manuscript at www.biomedcentral.com/submit
C Biomed Central 